\title{
Radiation-reaction-induced evolution of circular orbits of particles around Kerr black holes
}

\author{
Daniel Kennefick \\ Theoretical Astrophysics, California Institute of Technology, Pasadena, California 91125 \\ Amos Ori \\ Department of Physics, Technion - Israel Institute of Technology, Haifa 32000, Israel
}

(Received 30 November 1995)

\begin{abstract}
It is demonstrated that, in the adiabatic approximation, nonequatorial circular orbits of particles in the Kerr metric (i.e., orbits of constant Boyer-Lindquist radius) remain circular under the influence of gravitational radiation reaction. A brief discussion is given of conditions for breakdown of adiabaticity and of whether slightly noncircular orbits are stable against the growth of eccentricity.
\end{abstract}

PACS number(s): 04.30.Db, 04.25.Nx

\section{INTRODUCTION}

It has been shown some time ago that a particle in a circular orbit around a nonrotating black hole remains in a circular orbit under the influence of the gravitational radiation reaction arising from its orbital motion [1]. Although it has been suggested [2] that the same holds true for "circular" orbits (meaning orbits of constant Boyer-Lindquist radius [3]) around rotating black holes, up to now it has not been shown to beyond first post-Newtonian order (see Ref. [4] for the post-Newtonian result, and see also Refs. [5,6] for more recent work) because of the difficulty of dealing with the little understood "Carter" constant of the motion, $Q$. To date no practical method has been developed for describing the rate of change of this constant due to gravitational radiation reaction for a generic orbit (see, however, Ref. [7]), and without knowing this the evolution of the orbit cannot be predicted.

In this paper, we study the relation between the rates of change of the three constants of the orbital motion (the energy $E$, axial component of angular momentum $L$, and the Carter constant $Q$ ), for circular and almost-circular orbits. We first show that, for orbits which are precisely circular (in a sense which is well defined within the adiabatic approximation), the rate of change of $Q$ has just the value required for the orbit to evolve into a new circular orbit. Then, we extend the analysis to almost-circular orbits (to first order in the orbital eccentricity). This analysis leads to the result that in order for an initially circular orbit to develop nonzero eccentricity, the back-reaction force (evaluated for the precisely circular orbit) must resonate with the radial oscillations. Since in the case of a precisely circular orbit the periodicity of the back-reaction force is determined solely by the $\theta$ motion, we are led to the following conclusion. The only case in which an initially circular orbit will develop an eccentricity is when there is a certain resonance between the (small-oscillation) radial motion and the $\theta$ motion. More specifically, this resonance condition is $T_{\theta}=2 n T_{r}$, where $n$ is an integer, and $T_{r}$ and $T_{\theta}$ are the (averaged) periods of the radial motion and the angular motion, correspondingly.

Ryan [5] has recently examined circular orbits in the Kerr metric numerically and found that the above resonance condition is never satisfied (for all black-hole and orbital param- eters). We are thus led to the conclusion that orbits which are initially precisely circular will remain circular upon radiation-reaction evolution. We point out, however, that this conclusion does not address the issue of stability against the growth of a small initial eccentricity (this issue is further discussed in Sec. V below).

This paper is organized as follows. In Sec. II we define instantaneous circularity of an orbit in terms of the instantaneous location and four-velocity of the orbiting particle. We then derive a relation between the rates of change of $E, L$, and $Q$ for an instantaneously circular orbit that is perturbed by an arbitrary force and we show that this relation is precisely the one required for the circular orbit to evolve into a new circular orbit. One might naively interpret this result by itself as implying that an initially circular orbit will necessarily remain circular. However, in Sec. III we show that in order to predict the full evolution of initially circular orbits, it is necessary to carry the analysis (of the relation between the rates of change of the three constants of motion) to first order in the instantaneous eccentricity. This analysis, to first order in the eccentricity, is presented in Sec. IV, with the conclusion that initially circular orbits do, indeed, remain circular. Finally, in Sec. V we give some concluding remarks.

\section{INSTANTANEOUSLY CIRCULAR ORBITS}

In this section we shall define instantaneous circularity of an orbit and shall show that for any such orbits $d Q / d \tau$ has just the right value so as to leave the evolving orbit circular. We shall show that this is true for any arbitrary force which acts on the orbiting particle (in fact, this result is precise, and is not limited to the adiabatic approximation).

We take here the point of view that the radiative evolution may be viewed as the consequence of some "back-reaction force," which can be treated as any other external force [8]. We shall therefore begin by constructing a general formal expression for the evolution rate of all constants of (geodesic) motion, due to an arbitrary external force. Let $C$ denote the constant of motion in question. In Kerr, $C$ may stand for either the energy $E$, the azimuthal angular momentum $L$, or the Carter constant $Q$ (or any combination of these constants, like, e.g., the constant $D$ defined below). We first express all these constants explicitly as functions of coordinates and co- 
variant components of four-velocity [9]: that is,

$$
C \equiv C\left(x^{\beta}, u_{\alpha}\right) .
$$

For $E$ and $L$, we simply take

$$
E=-u_{t}, \quad L=u_{\varphi} .
$$

(Throughout, we use the standard Boyer-Lindquist coordinates, $r, t, \theta, \varphi$.) The corresponding explicit expression for $Q$ is slightly more complicated. We could use the familiar expression based on the $\theta$ motion in Kerr:

$$
Q=u_{\theta}^{2}+\cos ^{2} \theta\left[a^{2}\left(1-u_{t}^{2}\right)+\sin ^{-2} \theta u_{\varphi}^{2}\right] .
$$

We find it more convenient, however, to construct the expression for $Q$ from the $r$ motion. It is straightforward to show that

$$
Q=\Delta^{-1}\left[E\left(r^{2}+a^{2}\right)-a L\right]^{2}-(L-a E)^{2}-r^{2}-\Delta u_{r}^{2}
$$

[this follows, for instance, from Eqs. (33.32b) and (33.33c) in Ref. [10]]. Here, $\Delta \equiv r^{2}-2 M r+a^{2}$, where $M$ is the black hole's mass and $a M$ is its angular momentum. For later convenience, we also write this equation in the form

$$
Q=H(r, E, L)-\Delta u_{r}^{2},
$$

where

$H(r, E, L) \equiv \Delta^{-1}\left[E\left(r^{2}+a^{2}\right)-a L\right]^{2}-(L-a E)^{2}-r^{2}$.

In view of Eq. (2), one readily sees that Eq. (4) is just of the desired form, $Q=Q\left(x^{\beta}, u_{\alpha}\right)$ (with the simplification that the right-hand side does not depend on $\theta$ or $u_{\theta}$ ).

When an external force is applied to the particle, $C$ will evolve with time. To calculate its rate of change, we differentiate Eq. (1) with respect to the proper time $\tau$ :

$$
\frac{d C}{d \tau}=u^{\beta} C_{, \beta}+\sum_{\alpha} \frac{\partial C}{\partial u_{\alpha}} \frac{d u_{\alpha}}{d \tau} .
$$

Now,

$$
\frac{d u_{\alpha}}{d \tau}=\frac{D u_{\alpha}}{D \tau}+\frac{1}{2} g_{\mu \nu, \alpha} u^{\mu} u^{\nu}
$$

and

$$
\frac{D u_{\alpha}}{D \tau}=F_{\alpha}
$$

where $D / D \tau$ denotes covariant proper-time differentiation and $F_{\alpha}$ is the force per unit rest mass. Equation (7) thus reads

$\frac{d C}{d \tau}=\left[u^{\beta} C_{, \beta}+\frac{1}{2} \sum_{\alpha} \frac{\partial C}{\partial u_{\alpha}} g_{\mu \nu, \alpha} u^{\mu} u^{\nu}\right]+\sum_{\alpha} \frac{\partial C}{\partial u_{\alpha}} F_{\alpha}$.

Notice that the term in brackets does not depend on the external force. When no external force is applied, $C$ is conserved. Thus, the term in brackets must vanish identically. Equation (10) therefore reads

$$
\frac{d C}{d \tau}=\sum_{\alpha} \frac{\partial C}{\partial u_{\alpha}} F_{\alpha} .
$$

This is the desired general expression for the evolution rate of all constants of motion. Note that this is the precise expression for the instantaneous rate of change of $C$. We have not used the adiabatic approximation (or any other approximation) so far.

We now define an orbit to be instantaneously circular if its instantaneous values of $E, L$, and $Q$ [defined by Eqs. (2) and (5)] are precisely equal to those of some circular geodesic orbit. At a moment when the orbit (on which the force $F_{\alpha}$ acts) is instantaneously circular, Eq. (4) plus circularity $\left(u_{r}=0\right)$ implies

$$
\frac{\partial Q}{\partial u_{r}}=-2 \Delta u_{r}=0 .
$$

Inserting this into Eq. (11) gives

$$
\frac{d Q}{d \tau}=\frac{\partial Q}{\partial u_{t}} F_{t}+\frac{\partial Q}{\partial u_{\varphi}} F_{\varphi} .
$$

Also, in view of Eq. (2), Eq. (11) yields

$$
\frac{d E}{d \tau}=-F_{t}, \quad \frac{d L}{d \tau}=F_{\varphi} .
$$

Substituting Eqs. (2) and (14) in Eq. (13), we obtain

$$
\frac{d Q}{d \tau}=Q_{, E} \frac{d E}{d \tau}+Q_{, L} \frac{d L}{d \tau} .
$$

Finally, using Eq. (5), we rewrite Eq. (15) as

$$
\frac{d Q}{d \tau}=H_{, E} \frac{d E}{d \tau}+H_{, L} \frac{d L}{d \tau} .
$$

This is our final expression for the actual, momentary, rate of change of $Q$ due to the external force. [To avoid confusion, we emphasize that the partial derivatives on the right-hand side of Eq. (16) are to be calculated according to the explicit expression (6).]

We come now to the second part of this calculation, that is to calculate the rate of change of $Q$ (compared to that of $E$ and $L$ ) required for taking a circular orbit into a new circular orbit. From Eq. (5) we obtain

$$
\Delta u_{r}^{2}=H(r, E, L)-Q \equiv W(r, E, L, Q) .
$$

When applied to general geodesic orbits (with constant but arbitrary $E, L$, and $Q$ ) this equation can be regarded as describing radial motion in an effective potential $W(r, E, L, Q)$. Obviously that geodesic motion is circular if and only if the particle sits at a radius $r$ where $W=0$ (or $u_{r}=0$ ) and where $W_{, r}=0$ (so the particle is at the minimum of the effective potential). Correspondingly, an orbit on which a force $F_{\alpha}$ acts is instantaneously circular if and only if it instantaneously satisfies

$$
W=0, \quad W_{, r}=0 .
$$


We now let the force $F_{\alpha}$ continue to act, but only for an infinitesimal proper time, $\delta \tau$. Following this action, the orbit will be characterized by new parameters, $r^{\prime}, E^{\prime}, L^{\prime}$, and $Q^{\prime}$. We denote the changes from the original parameters by $\delta$, that is, $\delta r=r-r^{\prime}, \delta E=E-E^{\prime}$, etc. These changes are infinitesimal because $F_{\alpha}$ is allowed to act for only an infinitesimal time $\delta \tau$, before the orbit is once more examined for circularity. The corresponding change in $W$ is given by

$$
\delta W=W_{, r} \delta r+W_{, E} \delta E+W_{, L} \delta L+W_{, Q} \delta Q .
$$

In order for Eq. (18) to hold after the time $\delta \tau$ as well as before (i.e., in order for the orbit to remain circular), we must demand

$$
\delta W=0 .
$$

We denote the value of $Q$ which corresponds to a circular orbit (for given $E$ and $L$ ) by $Q_{\text {circ }}(E, L)$. Equations (19) and (20) thus yield, as a necessary condition for the orbit to remain circular

$$
W_{, r} \delta r+W_{, E} \delta E+W_{, L} \delta L+W_{, Q} \delta Q_{\text {circ }}=0,
$$

from which $\delta Q_{\text {circ }}$ is to be determined. Now Eq. (17) implies

$$
W_{, Q}=-1, \quad W_{, E}=H_{, E}, \quad W_{, L}=H_{, L},
$$

which together with $W_{, r}=0$ [Eq. (18)] reduces Eq. (21) to the form [11]

$$
\delta Q_{\text {circ }}=H_{, E} \delta E+H_{, L} \delta L .
$$

Finally, dividing by the infinitesimal proper time lapse $\delta \tau$ and taking the limit $\delta \tau \rightarrow 0$, we obtain

$$
\frac{d Q_{\text {circ }}}{d \tau}=H_{, E} \frac{d E}{d \tau}+H_{, L} \frac{d L}{d \tau} .
$$

Comparing Eqs. (16) and (24), we arrive at the desired result. At any moment when the orbit, on which the arbitrary force $F_{\alpha}$ acts, is instantaneously circular, $F_{\alpha}$ produces an instantaneous evolution of the orbit's Cater constant given by

$$
\frac{d Q}{d \tau}=\frac{d Q_{\mathrm{circ}}}{d \tau},
$$

which maintains instantaneous circularity.

For later convenience, let us define

$$
D \equiv Q_{\text {circ }}-Q=D(E, L, Q) .
$$

Circular orbits are thus characterized by $D=0$. Equation (25) then implies that if at a particular moment $D=0$, then

$$
d D / d \tau=0 .
$$

It should be emphasized again that all the calculations done so far are precise, and do not depend on the adiabatic approximation. Note also that these calculations refer to the instantaneous rate of change of the constants of motion, at a moment when the orbit is instantaneously circular. As we shall see in the next section, Eq. (27) by itself does not imply that an initially circular orbit will necessarily remain circular. One must go to the next order in the eccentricity in order to derive this result.

\section{THE NEED FOR ECCENTRICITY CORRECTIONS}

Equation (27) (which holds whenever $D=0$, i.e., whenever the orbit is instantaneously circular) has a trivial exact solution, $D(\tau)=0$. Does this necessarily mean that an instantaneously circular orbit will remain circular forever? We shall immediately see that, in principle, the answer is no (though, we shall also see later that, within the adiabatic approximation, in most cases a circular orbit will remain circular). In fact, the trivial solution $D(\tau)=0$ to Eq. (27) is physically meaningless.

To illustrate this, consider the analogous (but much simpler) problem of a free particle in one-dimensional Newtonian mechanics. The particle's energy (per unit rest mass) is $K=(1 / 2)(d x / d \tau)^{2}$. Assume now that a constant external force $F$ (per unit rest mass) is applied on the particle. The evolution of $K$ is then given by [in analogy with Eq. (11)]

$$
\frac{d K}{d \tau}=F \frac{d x}{d \tau},
$$

or, in terms of $K$ itself, by

$$
\frac{d K}{d \tau}=\sqrt{2} F K^{1 / 2} .
$$

Now, assume that the particle is initially at rest, i.e., $K=0$. Then, from Eq. (29), $d K / d \tau=0$. Equation (29) then admits a trivial exact solution,

$$
K(\tau)=0 .
$$

This trivial solution, which means that the particle will remain at rest forever, is obviously wrong. Instead, the particle will certainly accelerate, and the true physical solution is [12]

$$
K=\left(F^{2} / 2\right) \tau^{2} .
$$

The situation here with respect to Eq. (27) and its unphysical solution $D(\tau)=0$ is just analogous. As will be shown below (Sec. IV), in the extension of Eq. (27) to slightly eccentric orbits, $d D / d \tau$ is (to the leading order) proportional to $D^{1 / 2}$. Then, in addition to the trivial solution $D(\tau)=0$, there exists a nontrivial solution in which for some short period $D$ grows like $\tau^{2}$ [analogous to Eq. (31)], and this is the physical solution. This implies that, momentarily, the eccentricity (which is proportional to $D^{1 / 2}$ ) will grow linearly with $\tau$, even if initially it vanishes precisely.

Recall, however, that we are not particularly interested here in the momentary rate of change at a specific point along the orbit. Rather, we are interested in the effective long-term evolution of $D$. Within the adiabatic approximation, this long-term evolution is described by an equation of the form

$$
\dot{D}=S(E, L, D) \text {. }
$$


Hereafter, an overdot denotes the long-term rate of change (with respect to the proper-time, $\tau$ ), obtained from the momentary equation of motion by averaging over many periods (in Sec. IV we shall describe this averaging procedure in more detail). The long-term evolution of circular orbits will depend on the asymptotic behavior of $S$ near $D=0$. It is not difficult to show, based on Eq. (27), that the zero-order term [i.e., $S(D=0)]$ vanishes identically [13]. As we shall see in Sec. IV below, the general asymptotic form of $S$ is given by

$$
S(E, L, D)=S_{1}(E, L) D^{1 / 2}+S_{2}(E, L) D+O\left(D^{3 / 2}\right) .
$$

The evolution of an instantaneously circular orbit will depend crucially on whether $S_{1}$ vanishes or not. In the case $S_{1}=0$, we can approximate Eq. (33) by the linear equation

$$
\dot{D}=S_{2} D
$$

Then, an initial value $D=0$ ensures that $D$ will remain zero forever (we are not concerned here about stability to small initial perturbations, though we shall discuss the question of stability briefly in Sec. V). On the other hand, if $S_{1}$ is nonzero, we can approximate Eq. (33) by

$$
\dot{D}=S_{1} D^{1 / 2}
$$

As was explained above, the trivial solution $D(\tau)=0$ is physically meaningless. In that case, the physical solution will be (at least as long as $S_{1}>0$ )

$$
D(\tau)=\left(S_{1} / 2\right)^{2} \tau^{2}
$$

which means that the an instantaneously circular orbit will evolve into an eccentric one.

The above considerations make it clear that the value of $S_{1}$ is crucial for our discussion. An orbit which is initially instantaneously circular will (in the adiabatic approximation) remain circular if and only if $S_{1}=0$. In the next section we shall calculate $S_{1}$, and show that it generically vanishes. Only orbits which satisfy a certain resonance condition may have nonzero $S_{1}$.

\section{SLIGHTLY ECCENTRIC ORBITS}

We now analyze the adiabatic evolution of $D$ for slightly eccentric orbits, to leading order in the eccentricity. In order to use Eq. (11) for the calculation of $d D / d \tau$, we must first express $D$ in the form $D\left(x^{\beta}, u_{\alpha}\right)$. To simplify the notation, we make use of Eq. (2), and simply write $L$ instead of $u_{\varphi}$ and $-E$ instead of $u_{t}$. Recalling that $Q_{\text {circ }} \equiv Q_{\text {circ }}(E, L)$, we have, from Eq. (26),

$$
D=Q_{\text {circ }}(E, L)-Q\left(r, u_{r}, E, L\right)=D\left(r, u_{r}, E, L\right) .
$$

Here, $Q\left(r, u_{r}, E, L\right)$ is to be understood in terms of Eq. (4). This function is analytic in its arguments, and it is also not difficult to show that (except perhaps at the "last stable circular orbit," which must be excluded here), $Q_{\text {circ }}(E, L)$ is analytic in $E$ and $L$. Therefore, $D\left(r, u_{r}, E, L\right)$ in Eq. (37) is also analytic in its arguments. Now, Eq. (11) yields

$$
\begin{aligned}
\frac{d D}{d \tau} & =-D_{, E} F_{t}+D_{, L} F_{\varphi}+D_{, u_{r}} F_{r} \equiv D^{t} F_{t}+D^{\varphi} F_{\varphi}+D^{r} F_{r} \\
& =D^{j} F_{j},
\end{aligned}
$$

where the index $j$ runs over the three coordinates $r, t, \varphi$, and

$$
D^{j} \equiv \partial D / \partial u_{j}
$$

Equation (38) [like Eq. (11)] describes the precise, instantaneous rate of change of $D$, for any orbit on which any force $F_{\alpha}$ is acting. We have not made use of the adiabatic (or any other) approximation so far.

From the analyticity of $D\left(r, u_{r}, E, L\right)$ it follows that the functions

$$
D^{j} \equiv D^{j}\left(r, u_{r}, E, L\right)
$$

are analytic in their arguments. Now, from the validity of Eq. (27) for an external force of any type, it follows that for an instantaneously circular orbit [i.e., when $u_{r}=0$ and $\left.r=r_{\text {circ }}(E, L)\right]$, all three functions $D^{j}$ vanish. In other words, for any given $E$ and $L$,

$$
D^{j}\left(r=r_{0}, u_{r}=0, E, L\right)=0,
$$

where $r_{0} \equiv r_{\text {circ }}(E, L)$. We now expand $D^{j}\left(r, u_{r}, E, L\right)$ around $\left(r=r_{0}, u_{r}=0\right)$. In view of the analyticity of these functions, we have

$D^{j}\left(r, u_{r}, E, L\right)=\delta r D_{, r}^{j}+u_{r} D_{, u_{r}}^{j}+O\left(\delta r^{2}, u_{r}^{2}, u_{r} \delta r\right)$,

where $\delta r \equiv r-r_{0}$, and the functions $D_{, r}^{j}$ and $D_{, u_{r}}^{j}$ (which, again, are analytic functions of $\left.r, u_{r}, E, L\right)$ are evaluated at $\left(\delta r=0, u_{r}=0\right)$.

We are now going to use two approximations (or expansions): the adiabatic approximation, and the smalleccentricity approximation. These two approximations are unrelated, and should not be confused with each other. The exact instantaneous equation of motion of $D$ is [cf. Eq. (38)]

$$
\frac{d D}{d \tau}=D^{j} F_{j}
$$

The adiabatic approximation means that the external force $F_{j}$ is assumed to be small, and the right-hand side is to be evaluated to linear order in it. The small-eccentricity approximation means that the eccentricity is assumed small and Eq. (43) is evaluated to first order in it.

In view of the small-eccentricity approximation, the higher-order terms on the right-hand side of Eq. (42) can be omitted. Substitution in Eq. (43) then yields

$$
\frac{d D}{d \tau}=\left[\delta r(\tau) D_{, r}^{j}+u_{r}(\tau) D_{, u_{r}}^{j}\right] F_{j}(\tau) .
$$

Let us now examine the implications of the two approximations used here on the expression on the right-hand side. In view of the adiabatic approximation, the term in brackets is to be evaluated as if the constants of motion are fixed and the motion is geodesic. In the most general case, $D_{, r}^{j}$ and $D_{, u_{r}}^{j}$ are (like $\left.D\right)$ functions of $\left(r, u_{r}, E, L\right)$. Here, due to the 
adiabatic approximation, we can fix $E$ and $L$. Moreover, since $\delta r$ and $u_{r}$ are already first order in the eccentricity, when evaluating $D_{, r}^{j}$ and $D_{, u_{r}}^{j}$ we may take $u_{r}=0$ and $r=r_{0}$. Thus, in Eq. (44), $D_{, r}^{j}$ and $D_{, u_{r}}^{j}$ are just constants (which depend parametrically on $E$ and $L$ ) [14].

Turn now to evaluate $\delta r(\tau)$ and $u_{r}(\tau)$ on the right-hand side of Eq. (44). Like the entire term in brackets, they are to be evaluated as if the motion is geodesic (with fixed $E, L, D)$. In view of the small-eccentricity approximation, we need only calculate $\delta r(\tau)$ and $u_{r}(\tau)$ to the leading order in the eccentricity. We start from the "effective-potential" relation

$$
\Delta u_{r}^{2}=W(r, E, L, Q)
$$

[cf. Eq. (17)], and, recalling that $g^{r r}=g_{r r}^{-1}=\Delta / \rho^{2}$, we write it as

$$
(d r / d \tau)^{2}=\left(\Delta^{2} / \rho^{4}\right) u_{r}^{2}=\rho^{-4} W \Delta .
$$

Here,

$$
\rho^{2} \equiv r^{2}+a^{2} \cos ^{2} \theta
$$

In Eq. (46), as it stands, the radial motion is coupled to the $\theta$ motion, through $\rho$. In order to decouple the two motions, we define a new independent variable $\lambda$ by

$$
d \lambda / d \tau=\rho^{-2} .
$$

The radial equation of motion now becomes

$$
(d r / d \lambda)^{2}=W \Delta,
$$

in which the right-hand side is independent of $\theta$.

From Eqs. (17) and (6), $W$ is an analytic function of $(r, E, L, Q)$. Writing $Q=Q_{\text {circ }}(E, L)-D$, and recalling the analyticity of $Q_{\text {circ }}(E, L), W$ can be expressed as an analytic function of $(r, E, L, D)$. For an instantaneously circular orbit (i.e., for $r=r_{0}$ and $D=0$ ), both $W$ and $W_{, r}$ vanish [cf. Eq. (18)]. The expansion of $W$ near instantaneous circularity must therefore be of the form

$$
W=\hat{A} D+\hat{B} \delta r^{2}+O\left(D^{2}, D \delta r, \delta r^{3}\right),
$$

where $\hat{A}$ and $\hat{B}$ are some functions of $E$ and $L$. Correspondingly, the expansion of the right-hand side of Eq. (49) will take the form

$$
W \Delta=A D-B \delta r^{2}+O\left(D^{2}, D \delta r, \delta r^{3}\right),
$$

where $A \equiv \hat{A} \Delta_{0}$ and $B \equiv-\hat{B} \Delta_{0}$, and where

$$
\Delta_{0} \equiv \Delta\left(r=r_{0}(E, L)\right) .
$$

Combining Eqs. (49) and (51), and restricting attention to the leading-order eccentricity effect, we obtain

$$
(d \delta r / d \lambda)^{2}=A D-B \delta r^{2} .
$$

Note that $A$ and $B$ are some functions of $E$ and $L$ only. Therefore, as explained above, they can be regarded here as fixed parameters.
Equation (53) describes a simple harmonic oscillator. Its general solution is

$$
\delta r(\lambda)=K \sqrt{D} \cos \left[\omega_{r}\left(\lambda-\lambda_{0}\right)\right],
$$

where $K=\sqrt{A / B}$ and $\omega_{r}=\sqrt{B}$ are parameters that depend on $E$ and $L$ only. (Do not confuse $K$ here with $K$ of Sec. III.) Using this result to calculate $u_{r}$, we find

$$
u_{r}=g_{r r} \frac{d r}{d \tau}=\Delta^{-1} \frac{d \delta r}{d \lambda}=-\Delta^{-1} K \sqrt{D} \omega_{r} \sin \left[\omega_{r}\left(\lambda-\lambda_{0}\right)\right] .
$$

To simplify the notation, we shall hereafter absorb the constant $\lambda_{0}$ into $\lambda$ (by shifting the origin of the latter if necessary). Substituting Eqs. (54) and (55) into Eq. (44), we obtain

$$
\frac{d D}{d \tau}=\left\{K \sqrt{D}\left[D_{, r}^{j} \cos \left(\omega_{r} \lambda\right)-D_{,{ }_{r}}^{j} \Delta^{-1} \omega_{r} \sin \left(\omega_{r} \lambda\right)\right]\right\} F_{j}(\tau) .
$$

From Eq. (56) it is already clear that, so far as the instantaneous evolution is concerned, $d D / d \tau$ is indeed proportional to $\sqrt{D}$. Thus, as explained in Sec. III, although $D=0$ yields $d D / d \tau=0$, an instantaneously circular orbit will not remain circular later on. Instead, a momentary growth of $D$ like $\tau^{2}$ is to be anticipated. However, we are primarily interested here in the long-term adiabatic evolution of $D$. The latter is to be obtained from Eq. (56) by averaging it in $\tau$ over many periods. In order to perform this long-term averaging, we must first take a closer look at the nature of the time dependence of the force $F_{j}$.

Since the term in brackets on the right-hand side of Eq. (56) is already proportional to $\sqrt{D}$ (i.e., to the eccentricity), when evaluating $F_{j}(\tau)$ we are allowed to assume that the orbit is a precisely circular geodesic. The $\theta$ motion of such an orbit is periodic in $\tau$. Therefore, the backreaction force must be periodic also. The various points along the circular geodesic orbit are physically distinguishable from one another only by the values of $\theta$ and $d \theta / d \tau$. It therefore follows that after completing a full cycle of the $\theta$ motion, $F_{j}(\tau)$ will return to its original value. A closer look, however, reveals that, because of the reflection symmetry of the Kerr geometry, the $\theta$ motion is symmetric with respect to the equatorial plane. As a consequence, the period of $F_{j}(\tau)$ will in fact be half of that of the full $\theta$-motion cycle.

In order to facilitate the calculations, it is convenient to transform Eq. (56) from $\tau$ to $\lambda$. Using Eq. (48), we obtain

$$
\begin{aligned}
\frac{d D}{d \lambda}= & K \sqrt{D}\left[\rho ^ { 2 } \left(D_{, r}^{j} \cos \left(\omega_{r} \lambda\right)\right.\right. \\
& \left.\left.-D_{,{ }_{, r}}^{j} \Delta^{-1} \omega_{r} \sin \left(\omega_{r} \lambda\right)\right)\right] F_{j}(\tau(\lambda)) .
\end{aligned}
$$

According to our expansion scheme, we need only evaluate the term in brackets to zero order in the eccentricity. That is, we can replace $\rho$ and $\Delta$ by their circular counterparts, $\rho_{0}$ and $\Delta_{0}$, where $\Delta_{0}$ is the constant defined in Eq. (52), and $\rho_{0}$ is a function of $\theta$ only, defined by

$$
\rho_{0}^{2} \equiv r_{0}^{2}+a^{2} \cos ^{2} \theta .
$$


We therefore obtain

$$
\frac{d D}{d \lambda}=K \sqrt{D}\left[D_{, r}^{j} \cos \left(\omega_{r} \lambda\right)-D_{,{ }_{r}}^{j} \Delta_{0}^{-1} \omega_{r} \sin \left(\omega_{r} \lambda\right)\right]\left[\rho_{0}^{2} F_{j}\right] .
$$

The term in brackets depends on $\tau$ (and hence on $\lambda$ ) through its dependance on the $\theta$ motion. It is obvious from Eq. (58) that $\rho_{0}$ is periodic in $\tau$, again with a period which is just half that of the $\theta$ cycle. Consequently, the entire term in brackets is also periodic (with that one-half $\theta$ period). Let us examine now the periodicity of this term with respect to $\lambda$. Again, the $\theta$ motion is periodic in $\lambda$, and the reflection symmetry implies that the motion at $\theta<\pi / 2$ is just symmetric to that at $\theta>\pi / 2$. [This can also be deduced directly from the fact that, in the equation of motion for $\theta(\lambda),(d \theta / d \lambda)^{2}=\Theta(\theta)$ (cf. Ref. [10]), the function $\Theta(\theta)$ admits a reflection symmetry about $\pi / 2$.] Thus, if we denote the $\lambda$ period of the $\theta$ motion by

$$
\Lambda_{\theta} \equiv 2 \pi / \omega_{\theta},
$$

then the term in brackets in Eq. (59) has a $\lambda$ period of $\Lambda_{\theta} / 2$. Correspondingly, the Fourier transform of this term will take the form

$$
\rho_{0}^{2} F_{j}=\sum_{n=0}^{\infty} G_{j}^{n} e^{i 2 n \omega_{\theta} \lambda}+\text { c.c. }
$$

where c.c. means the complex conjugate of the preceding term.

Substituting this expansion in Eq. (59) yields

$$
\begin{aligned}
\frac{d D}{d \lambda}= & \sqrt{D}\left[K \left(D_{, r}^{j} \cos \left(\omega_{r} \lambda\right)\right.\right. \\
& \left.\left.-D_{,{ }_{r}}^{j} \Delta_{0}^{-1} \omega_{r} \sin \left(\omega_{r} \lambda\right)\right)\right] \sum_{n=0}^{\infty} G_{j}^{n} e^{2 n i \omega_{\theta} \lambda}+\text { c.c. }
\end{aligned}
$$

It is convenient to transform the last expression from sine and cosine to exponential functions. The term in brackets can be expressed as

$$
K_{+}^{j} e^{i \omega_{r} \lambda}+K_{-}^{j} e^{-i \omega_{r} \lambda}
$$

where

$$
K_{ \pm}^{j} \equiv(K / 2)\left(D_{, r}^{j} \pm i D_{,{ }_{r}}^{j} \Delta_{0}^{-1} \omega_{r}\right) .
$$

Equation (62) then takes the form

$$
\frac{d D}{d \lambda}=\sqrt{D}\left(K_{+}^{j} e^{i \omega_{r} \lambda}+K_{-}^{j} e^{-i \omega_{r} \lambda}\right) \sum_{n=0}^{\infty} G_{j}^{n} e^{2 n i \omega_{\theta} \lambda}+\text { c.c. }
$$

Defining now

$$
G_{ \pm}^{n} \equiv K_{ \pm}^{j} G_{j}^{n}
$$

we obtain

$$
\frac{d D}{d \lambda}=\sqrt{D} \sum_{n=0}^{\infty}\left(G_{+}^{n} e^{i\left(2 n \omega_{\theta}+\omega_{r}\right) \lambda}+G_{-}^{n} e^{i\left(2 n \omega_{\theta}-\omega_{r}\right) \lambda}\right)+\text { c.c. }
$$

Recall that in this equation the coefficients $G_{ \pm}^{n}$ depend on $E$ and $L$, but not on $\lambda$.

Equation (67) describes (within the adiabatic approximation, and to leading order in the eccentricity) the instantaneous rate of change of $D$. In order to obtain from it the long-term rate of change, we simply need to take its average over a sufficiently long period of $\lambda$. To that end, for any function $U(\lambda)$, we formally define the long-term averaged rate of change

$$
\left\langle\frac{d U}{d \lambda}\right\rangle \equiv \lim _{\Delta \lambda \rightarrow \infty} \frac{\Delta U}{\Delta \lambda}
$$

where $\Delta U$ and $\Delta \lambda$ denote the difference in $U$ and $\lambda$, correspondingly, between the two extremes of the $\lambda$ interval considered. Although the averaging is over times long compared to $1 / \omega_{\theta}$ and $1 / \omega_{r}$, it is still short compared to the radiation reaction time scale, which is the time for substantial orbital inspiral. (Recall that since we are using the adiabatic approximation here, if $U$ also depends on the "constants of motion," they must be taken as fixed constants in this averaging process.) The averaging of the right-hand side of Eq. (67) is trivial, in that the term $e^{i\left(2 n \omega_{\theta}^{ \pm} \omega_{r}\right) \lambda}$ will average to zero, unless $2 n \omega_{\theta} \pm \omega_{r}=0$, in which case it averages to one. Since both $\omega_{\theta}$ and $\omega_{r}$ are taken to be positive, we need only worry about those terms with $2 n \omega_{\theta}-\omega_{r}$ in the exponent. We thus obtain

$$
\left\langle\frac{d D}{d \lambda}\right\rangle=\sqrt{D} \sum_{n=0}^{\infty} G_{n} \delta_{\left(2 n \omega_{\theta}-\omega_{r}\right)},
$$

where $\delta$ is a function whose value is unity when $2 n \omega_{\theta}-\omega_{r}=0$ and zero otherwise, and $G_{n} \equiv 2 \operatorname{Re}\left(G_{-}^{n}\right)$, with Re meaning the "real part of."

At this stage it is already clear that, unless a certain resonance condition is satisfied $\left(\omega_{r}=2 n \omega_{\theta}\right.$ for some $\left.n\right)$, the right-hand side of Eq. (69) will vanish. Before we further discuss the meaning and implications of this resonance condition, however, we shall more directly connect our result (69) to the notation used in Sec. III, and in particular to the parameter $S_{1}$. Equation (35) is to be obtained from the momentary rate of change of $D$ by averaging over proper time. For any function $U(\tau)$, the long-term proper-time average (denoted by an overdot) may be formally defined as

$$
\dot{U} \equiv \lim _{\Delta \tau \rightarrow \infty} \frac{\Delta U}{\Delta \tau}=\lim _{\Delta \lambda \rightarrow \infty} \frac{\Delta \lambda}{\Delta \tau} \lim _{\Delta \lambda \rightarrow \infty} \frac{\Delta U}{\Delta \lambda}=J^{-1}\left\langle\frac{d U}{d \lambda}\right\rangle,
$$


where

$$
J \equiv\left\langle\frac{d \tau}{d \lambda}\right\rangle
$$

is a constant that depends on the orbit. Applying this procedure to $D$, we obtain [15]

$$
\dot{D}=J^{-1}\left\langle\frac{d D}{d \lambda}\right\rangle=\sqrt{D} J^{-1} \sum_{n=0}^{\infty} G_{n} \delta_{\left(2 n \omega_{\theta}-\omega_{r}\right)}
$$

Comparing now Eq. (72) to Eq. (35), we find

$$
S_{1}=J^{-1} \sum_{n=0}^{\infty} G_{n} \delta_{\left(2 n \omega_{\theta}-\omega_{r}\right)} .
$$

The implications of this result for the long-term evolution of $D$ are obvious. There are two different cases.

(a) The resonant case: there exists an integer $n$ such that $\omega_{r}=2 n \omega_{\theta}$. In that case, we have

$$
S_{1}=J^{-1} G_{n}
$$

which is likely to be nonzero in the general case. Then, as discussed in Sec. III [cf. Eq. (36)], $D$ will grow like $\tau^{2}$, which means that the eccentricity will grow linearly with $\tau$.

(b) The nonresonant case: there exists no integer $n$ for which $\omega_{r}=2 n \omega_{\theta}$. In that case, we simply have

$$
S_{1}=0
$$

and the equation of evolution will read

$$
\dot{D}=S_{2} D+O\left(D^{3 / 2}\right)
$$

[cf. Eq. (33)]. In this case an orbit which is initially precisely circular will remain circular (within the adiabatic limit). (The sign of $S_{2}$ will determine the stability against growth of small initial eccentricity.)

For resonant orbits, we have

$$
\Lambda_{\theta}=2 n \Lambda_{r}
$$

where $\Lambda_{\theta}$ and $\Lambda_{r}$ are the $\lambda$ periods of the $\theta$ and $r$ motions, correspondingly. It would sometimes be convenient to translate this expression to $t$ periods. One finds that, not surprisingly, the resonance condition is

$$
T_{\theta}=2 n T_{r}
$$

where $T_{\theta}$ is the $t$ period of the $\theta$ motion, and $T_{r}$ is the averaged $t$ period of the radial motions. [The radial motion, expressed in terms of $t$ (or $\tau$ ), is quasiperiodic rather than periodic, because it is modulated by the $\theta$ motion. The $\theta$ motion itself is periodic in either $t$ or $\tau$ - first, because we are considering a circular orbit here, and second, because the resonance condition (77) ensures that each time $\theta$ returns to its original value, $r$ does also (but not vice versa).]

\section{CONCLUSION}

We have shown that, within the adiabatic approximation, an orbit which is initially precisely circular will remain circular, under the action of the radiation-reaction force. The only exception is if the orbit satisfies the resonance condition $T_{\theta}=2 n T_{r}$, for some integer $n$, where $T_{\theta}$ is the $\theta$-motion period and $T_{r}$ is the (averaged) period of the smalloscillation radial motion. However, circular orbits around Kerr never satisfy this resonance condition [5]. We therefore conclude that, within the adiabatic approximation, an orbit which is initially circular will remain circular.

There are two caveats which should be mentioned here. First, no attempt has been made to address the issue of stability against the growth of a small initial eccentricity. This stability would depend on the sign of the coefficient $S_{2}$ in Eq. (33) above, which was not calculated here.

Second, our conclusion that circular orbits must remain circular is only valid within the adiabatic limit, i.e., in the limit $\mu / M \rightarrow 0$, where $\mu$ is the mass of the small object. In reality, since the ratio $\mu / M$ is always finite, an initially circular orbit will develop some eccentricity. For concreteness, consider a particle with $\mu \ll M$ which at some initial stage (which we denote stage 1) moves along a circular orbit with Boyer-Lindquist radius $r_{1}$. Later on, the orbit shrinks due to radiation reaction, until (at stage 2) it passes through a new radius, $r_{2}<r_{1}$. Then, for every finite $\mu / M$, one should expect nonzero eccentricity to be present at stage 2 . The above analysis, however, implies that the eccentricity at stage 2 will decrease with $\mu$ (for fixed $r_{1}, r_{2}$, and $M$ ), and will vanish at the limit $\mu / M \rightarrow 0$ (presumably like $\mu / M$ ) [16].

The small eccentricity of nonadiabatic origin mentioned above could in principle seed an exponential growth of eccentricity if $S_{2}>0$. In such a situation, an initially circular orbit may evolve into a very noncircular one (even for small $\mu / M)$. The feasibility of this scenario depends, of course, on the relevant values of $\mu / M$ and on whether $S_{2}>0$ and also on the available range of $r$ values (over which $S_{2}$ is positive).

In reality, we know that in the Newtonian limit the orbits become more and more circular as they shrink due to radiation reaction, so we expect $S_{2}$ to be negative as long as $r \gg M$ (recall that the Newtonian approximation should hold at $r \gg M$ even if the black hole is spinning). Consequently, the range over which $S_{2}$ might be positive is bounded. We can therefore expect that if $\mu / M$ is sufficiently small (and if $r_{2}$ is not too close to the "last stable circular orbit" [17]), the instability will not have enough time to build up, and an initially circular orbit will indeed remain circular throughout the inspiral, to a good approximation.

\section{ACKNOWLEDGMENTS}

We thank Kip Thorne, Fintan Ryan, Haris Apostolatos, and Eric Poisson for many helpful discussions. This research was supported in part by NASA Grant No. NAGW-4268 and by NSF Grant No. AST-9417371. 
[1] T. A. Apostolatos, D. Kennefick, A. Ori, and E. Poisson, Phys. Rev. D 47, 5376 (1993).

[2] This conjecture was made by one of us (A. Ori) several years ago.

[3] Such orbits have often been referred to as "quasicircular" in the literature (see, for instance, Ref. [10]), because such an orbit in Kerr does not describe a circle, or any closed curve and is not even confined, in general, to a single plane. However, for our purposes, the term circular aptly implies the analogy we wish to draw with the Keplerian orbit case (or the Schwarzschild case). That is, the orbit is described by a constant radial coordinate, and that characteristic is maintained under the influence of an adiabatic back reaction force.

[4] F. D. Ryan, Phys. Rev. D 52, R3159 (1995).

[5] An interesting qualitative argument is given in F. D. Ryan, Phys. Rev. D 53, 3064 (1996).

[6] Recently, we have become aware of an independent derivation of this result by Y. Mino, thesis in preparation, Kyoto University, Japan.

[7] A. Ori, Phys. Lett. A 202, 347 (1995).

[8] In the case of gravitational radiation reaction (which is our chief concern here), although it is possible to use this concept of back-reaction force, this force is gauge dependent. We do not expect this ambiguity to affect our results, for the following reason: despite the ambiguity in the momentary backreaction force, the long-term adiabatic evolution must be gauge independent. Therefore, one can simply pick any gauge in order to calculate the radiative evolution. Once the gauge is chosen, the back-reaction force is defined unambiguously, and all the analysis of this paper is applicable. Keep in mind, however, that since we will employ an argument based on the symmetries of Kerr, we must be careful to select a gauge which does not artificially violate these symmetries. The harmonic gauge is an example of a "good" gauge for our purposes.

[9] Note that this function $C\left(x^{\beta}, u_{\alpha}\right)$ is not unique. This is obvious, for example, from the fact that $-1=g^{\alpha \beta}(x) u_{\alpha} u_{\beta}$. The function $C\left(x^{\beta}, u_{\alpha}\right)$ can thus be chosen upon convenience.
[10] C. W. Misner, K. S. Thorne, and J. A. Wheeler, Gravitation (Freeman, New York, 1973).

[11] Taking the differential of the second condition in Eq. (18), $W_{, r}=0$, does not yield any additional constraint on $\delta Q_{\text {circ }}$ (instead it results in an expression for $\delta r_{\text {circ }}$ ).

[12] Mathematically, this ambiguity in the solution of Eq. (29) with the initial condition $K=0$ is possible due to the fact that the term $K^{1 / 2}$ on the right-hand side is not differentiable with respect to the unknown $K$. Note that this mathematical ambiguity does not reflect any ambiguity in the solution of the fundamental equation of motion (i.e., the Newton equation, $\left.d^{2} x / d \tau^{2}=F\right)$. The only ambiguity occurs in the equation for $K$ derived from $d^{2} x / d \tau^{2}=F$. The origin of this ambiguity is simple. In order to derive the equation for $K$, the fundamental equation of motion is multiplied by $d x / d \tau$, and this entity is identically zero in the trivial nonphysical solution (30). Thus, although the trivial solution (30) satisfies the equation for $K$, it does not satisfy the original equation of motion.

[13] This follows, for example, from Eq. (72) below.

[14] Of the six coefficients $D_{, r}^{j}$ and $D_{, u_{r}}^{j}$, three vanish identically $\left(D_{, r}^{r}, D_{, u_{r}}^{t}\right.$, and $\left.D_{, u_{r}}^{\varphi}\right)$ but the other three are generically nonzero.

[15] It is sometimes useful to have the averaged radiative evolution rates in terms of external time $t$ rather than $\tau$. The procedure for averaging over $t$ is analagous to the above averaging over $\tau$. The result is also similar. On the right-hand side of Eq. (72), one only needs to replace $J$ by the corresponding parameter, $J_{t} \equiv\langle d t / d \lambda\rangle$.

[16] The back-reaction force (per unit mass $\mu$ ) is proportional to $\mu$ (and the evolution time from stage 1 to stage 2 is proportional to $1 / \mu$ ). Now, while the force terms responsible for the adiabatic shrinking of the orbit from radius $r_{1}$ to $r_{2}$ are linear in the back-reaction force, the non-adiabatic effects are (at least) quadratic in that force. The nonadiabatic terms are therefore suppressed by the small factor $\mu / M$ (compared to the adiabatic terms).

[17] In the Schwarzschild case, it has been found [1] that $S_{2}$ diverges at the last stable circular orbit, due to the flattening of the effective potential well. Intuitively, one should expect the same behavior in Kerr as well. 